\title{
Boundary Value Methods for Second-Order PDEs via the Lanczos-Chebyshev Reduction Technique
}

\author{
T. A. Biala, ${ }^{1}$ S. N. Jator, ${ }^{2}$ and R. B. Adeniyi ${ }^{3}$ \\ ${ }^{1}$ Department of Mathematics and Computer Science, Sule Lamido University, PMB 048, Kafin Hausa, Nigeria \\ ${ }^{2}$ Department of Mathematics and Statistics, Austin Peay State University, Clarksville, TN 37044, USA \\ ${ }^{3}$ Department of Mathematics, University of Ilorin, Ilorin, Nigeria
}

Correspondence should be addressed to T. A. Biala; bialatoheeb@yahoo.com

Received 6 June 2016; Revised 11 December 2016; Accepted 9 January 2017; Published 30 January 2017

Academic Editor: Guillermo Botella-Juan

Copyright (C) 2017 T. A. Biala et al. This is an open access article distributed under the Creative Commons Attribution License, which permits unrestricted use, distribution, and reproduction in any medium, provided the original work is properly cited.

\begin{abstract}
In this paper, we study the performance of Boundary Value Methods (BVMs) on second-order PDEs. The PDEs are transformed into a system of second-order ordinary differential equations (ODEs) using the Lanczos-Chebyshev reduction technique. The conditions under which the BVMs converge and the computational complexities of the algorithms are discussed. Numerical illustrations are given to show the simplicity and high accuracy of the approach.
\end{abstract}

\section{Introduction}

The study of methods which approximates the solution of partial differential equations is an important issue in numerical analysis. This is because most modeled problems in science and engineering results in PDEs such as the problem of the elastic torsion of prismatic bars, axisymmetric ideal flow around sphere, the heat equation, the wave equations and so on. In this paper, we are interested in the approximate solution of the initial or boundary value problems of secondorder PDEs

$$
\mathbf{L} u=f,
$$

where $\mathbf{L}$ is a fixed elliptic or hyperbolic operator of order $2 m$, $f \in F$, and $F$ is a space function defined on a domain $D$.

The reduction of (1) to a lower dimensional problem results, in most cases, to either a system of second-order ordinary differential equation (ODE) or an algebraic system. The reduction technique described in this paper is based on the Lanczos $\tau$-method $[1,2]$. In this approach, polynomials are used as the trial functions with an important advantage that the "economized" solutions which can be readily integrated or differentiated are obtained $[1,2]$.

In the last few years, Boundary Value Methods (BVMs) have been used for the solution of the first-order initial and boundary value problems and their convergence and linear stability properties have been fully discussed in [3-8]. These BVMs are also used to solve higher order initial and boundary value problems by first reducing the higher order differential equations into an equivalent first-order system which increases the computational cost and time. Biala [9] and Biala et al. [10-12] developed BVMs for the direct solution of systems of the general second-order ODEs and PDEs with initial or boundary conditions. The boundary value technique simultaneously generates approximate solution $\left(y_{1}, y_{2}, \ldots, y_{N}\right)^{T}$ to the exact solution $\left(y\left(x_{1}\right), y\left(x_{2}\right), \ldots\right.$, $\left.y\left(x_{N}\right)\right)^{T}$ on the entire interval of integration. This approach has the advantage of producing smaller global errors (at the end of the range of integration) than those produced by the step-by-step methods due to the presence of accumulated errors at the each step in the step-by-step method. The novel property of this paper is in the ease with which the secondorder PDEs are transformed into a system of ODEs.

The paper is organized as follows: In Section 2, we derive a continuous approximation $U(x)$ of the exact solution $y(x)$ from which the class of BVMs are developed. The convergence and computational complexities of the methods are discussed in Section 3. In Section 4, the Lanczos-Chebyshev reduction technique is introduced. Several numerical illustrations, given in Section 5, are given to show the efficacy of 
this approach. Finally, we give a concluding remark in Section 6 .

\section{Boundary Value Methods}

The BVMs given in [9] are a class of methods for the general second-order ODEs based on the Linear Multistep Formulas. Most formulas [4] used for second-order ODEs are implemented by reducing them into a system of first-order ODEs. The BVMs in [9] were introduced in order to lessen the computational cost and time and also to utilize additional information associated with specific differential equations such as the oscillatory nature of solutions. One main feature of the BVMs in [9] is that they can be used in the same way for solving both initial and boundary value problems with some slight change in the code used. Therefore such methods are the best candidates for solving second-order PDEs after reduction into an equivalent second-order system.

In what follows, we consider the general system of second-order boundary value problems

$$
y^{\prime \prime}=f\left(x, y \cdot y^{\prime}\right), \quad x \in[a, b]
$$

subject to the mixed boundary conditions

$$
\begin{aligned}
& a_{0} y(a)+b_{0} y^{\prime}(a)=d_{o}, \\
& a_{1} y(b)+b_{1} y^{\prime}(b)=d_{1},
\end{aligned}
$$

where $f: \mathbb{R} \times \mathbb{R}^{2 m} \rightarrow \mathbb{R}^{m}$ are continuous functions, $y, y^{\prime}$, $y^{\prime \prime} \in \mathbb{R}^{m}$, and $m$ is the dimension of the system. The BVMs for solving (2) take the general form

$$
\begin{aligned}
y_{n+j}+\alpha_{v}^{(j)} y_{n+v}+\alpha_{0}^{(j)} y_{n}= & h^{2} \sum_{i=0}^{2 v} \beta_{i}^{(j)} f_{n+i}, \\
& \quad j=1, \ldots, v-1, v+1, \ldots, 2 v
\end{aligned}
$$

with the derivative formulas

$$
h y_{n+j}^{\prime}+\alpha_{v}^{\prime(j)} y_{n+v}+\alpha_{0}^{\prime(j)} y_{n}=h^{2} \sum_{i=0}^{2 v} \beta_{i}^{\prime(j)} f_{n+i},
$$

$$
j=0,1, \ldots, 2 v
$$

where $h$ is the constant stepsize and $\alpha_{v}^{(j)}, \alpha_{0}^{(j)}, \beta_{i}^{(j)}, \alpha_{v}^{\prime(j)}, \alpha_{0}^{\prime(j)}$, and $\beta_{i}^{(j)}$ are chosen so that (4) and (5) have order $2 v+2$ and $2 v+1$, respectively.

2.1. Derivation of the BVMs. In this section, we shall use the interpolation and collocation approach [9-15] to construct a $2 v$-step continuous LMM (CLMM) which will be used to produce formulas for solving (2). The CLMM has the general form

$$
U(x)=\alpha_{v}(x) y_{n+v}+\alpha_{0}(x) y_{n}+h^{2} \sum_{i=0}^{2 v} \beta_{i}(x) f_{n+i} .
$$

Evaluating (6) at $x_{j}, j=1, \ldots, v-1, v+1, \ldots, 2 v$ we obtain the formulas of the form

$$
\begin{aligned}
y_{n+j}+2 y_{n+v}-y_{n}=h^{2} \sum_{i=0}^{2 v} \beta_{i} f_{n+i}, & \\
& \quad j=1, \ldots, v-1, v+1, \ldots, 2 v
\end{aligned}
$$

whose derivative formula is

$$
h y_{n+k}^{\prime}+\alpha_{v}^{\prime} y_{n+v}+\alpha_{0}^{\prime} y_{n}=h^{2} \sum_{i=0}^{2 v} \beta_{i}^{\prime} f_{n+i}, \quad k=0(1)(2 v)
$$

To obtain (6), we seek an approximation $U(x)$ to the exact solution $y(x)$ of the form

$$
U(x)=\sum_{r=0}^{p+q-1} b_{r} x^{r}
$$

where $x \in[a, b]$ and $b_{r}$ are coefficients to be uniquely determined so that the method has order $2 v+2$ and $p$ and $q$ are the number of interpolation and collocation points, respectively. We impose that the interpolating function (9) satisfies the following conditions:

$$
\begin{aligned}
U\left(x_{v}\right) & =y_{v}, \\
U\left(x_{0}\right) & =y_{0}, \\
U^{\prime \prime}\left(x_{i}\right) & =f_{i}, \quad i=0(1)(2 v),
\end{aligned}
$$

which lead to a system of $2 v+3$ equations which is solved using a Computer Algebra System (CAS) such as Mathematica to obtain $b_{r}, r=0(1)(p+q-1)$.

The CLMM is developed by substituting the values of $b_{r}$ into (9). After some algebraic manipulations, the CLMM is expressed in form (6). The $2 v$-step CLMM is used to generate the main methods (7) and derivative formulas of form (8) which are combined to solve (2). For example, the BVMs of order 6 used are as follows.

BVM order 6:

$$
\begin{aligned}
& y_{n+1}-\frac{1}{2} y_{n+2}-\frac{1}{2} y_{n}=\frac{h^{2}}{480}\left(-19 f_{n}-204 f_{n+1}\right. \\
& \left.-14 f_{n+2}-4 f_{n+3}+f_{n+4}\right) \\
& y_{n+3}-\frac{3}{2} y_{n+2}+\frac{1}{2} y_{n}=\frac{h^{2}}{480}\left(17 f_{n}+252 f_{n+1}\right. \\
& \left.+402 f_{n+2}+52 f_{n+3}-3 f_{n+4}\right), \\
& y_{n+4}-2 y_{n+2}-y_{n}=\frac{h^{2}}{15}\left(f_{n}+16 f_{n+1}+26 f_{n+2}\right. \\
& \left.+16 f_{n+3}+f_{n+4}\right) \\
& \quad n=0(4)(N-4)
\end{aligned}
$$


with the derivative formulas

$$
\begin{aligned}
& h y_{n}^{\prime}-\frac{1}{2} y_{n+2}+\frac{1}{2} y_{n}=\frac{h^{2}}{180}\left(-53 f_{n}-144 f_{n+1}\right. \\
& \left.\quad+30 f_{n+2}-16 f_{n+3}+3 f_{n+4}\right), \\
& h y_{n+1}^{\prime}-\frac{1}{2} y_{n+2}+\frac{1}{2} y_{n}=\frac{h^{2}}{720}\left(39 f_{n}+70 f_{n+1}\right. \\
& \left.\quad-144 f_{n+2}+42 f_{n+3}-7 f_{n+4}\right), \\
& h y_{n+2}^{\prime}-\frac{1}{2} y_{n+2}+\frac{1}{2} y_{n}=\frac{h^{2}}{180}\left(5 f_{n}+104 f_{n+1}+78 f_{n+2}\right. \\
& \left.\quad-8 f_{n+3}+f_{n+4}\right), \\
& h y_{n+3}^{\prime}-\frac{1}{2} y_{n+2}+\frac{1}{2} y_{n}=\frac{h^{2}}{720}\left(31 f_{n}+342 f_{n+1}\right. \\
& \left.\quad+768 f_{n+2}+314 f_{n+3}-15 f_{n+4}\right), \\
& h y_{n+4}^{\prime}-\frac{1}{2} y_{n+2}+\frac{1}{2} y_{n}=\frac{h^{2}}{180}\left(3 f_{n}+112 f_{n+1}+56 f_{n+2}\right. \\
& \left.\quad+240 f_{n+3}+59 f_{n+4}\right) .
\end{aligned}
$$

Also, the BVMs of order 8 are as follows.

BVM order 8:

$$
\begin{aligned}
& y_{n+1}-\frac{1}{3} y_{n+3}-\frac{2}{3} y_{n}=\frac{h^{2}}{60480}\left(-2803 f_{n}-37950 f_{n+1}\right. \\
& \quad-14913 f_{n+2}-7108 f_{n+3}+3147 f_{n+4}-990 f_{n+5} \\
& \left.+137 f_{n+6}\right), \\
& y_{n+2}-\frac{2}{3} y_{n+3}-\frac{2}{3} y_{n}=\frac{h^{2}}{60480}\left(-1291 f_{n}-21906 f_{n+1}\right. \\
& \quad-32133 f_{n+2}-6288 f_{n+3}+1467 f_{n+4}-402 f_{n+5} \\
& \left.+53 f_{n+6}\right), \\
& y_{n+4}-\frac{4}{3} y_{n+3}+\frac{1}{3} y_{n}=\frac{h^{2}}{30240}\left(661 f_{n}+10734 f_{n+1}\right. \\
& +19323 f_{n+2}+27268 f_{n+3}+2523 f_{n+4}-18 f_{n+5} \\
& \left.\quad-11 f_{n+6}\right), \\
& y_{n+5}-\frac{5}{3} y_{n+3}+\frac{2}{3} y_{n}=\frac{h^{2}}{12096}\left(535 f_{n}+8550 f_{n+1}\right. \\
& +15501 f_{n+2}+22900 f_{n+3}+11889 f_{n+4}+1158 f_{n+5} \\
& \left.\quad-53 f_{n+6}\right), \\
& y_{n+6}-2 y_{n+3}+y_{n}=\frac{h^{2}}{2240}\left(141 f_{n}+2430 f_{n+1}\right. \\
& +4131 f_{n+2}+6756 f_{n+3}+4131 f_{n+4}+2430 f_{n+5} \\
& \left.+141 f_{n+6}\right)
\end{aligned}
$$$$
n=0(6)(N-6),
$$

with the derivatives

$$
\begin{aligned}
& h y_{n}^{\prime}-\frac{1}{3} y_{n+3}+\frac{1}{3} y_{n}=\frac{h^{2}}{13440}\left(-3795 f_{n}-14850 f_{n+1}\right. \\
& \quad+2403 f_{n+2}-6300 f_{n+3}+3267 f_{n+4}-1026 f_{n+5} \\
& \left.\quad+141 f_{n+6}\right), \\
& h y_{n+1}^{\prime}-\frac{1}{3} y_{n+3}+\frac{1}{3} y_{n}=\frac{h^{2}}{120960}\left(4019 f_{n}-3426 f_{n+1}\right. \\
& \quad-7125 f_{n+2}+18308 f_{n+3}-11019 f_{n+4}+3390 f_{n+5}
\end{aligned}
$$

$\left.-457 f_{n+6}\right)$,

$$
\begin{aligned}
& h y_{n+2}^{\prime}-\frac{1}{3} y_{n+3}+\frac{1}{3} y_{n}=\frac{h^{2}}{120960}\left(2293 f_{n}\right. \\
& \quad+46830 f_{n+1}+22683 f_{n+2}-14204 f_{n+3}+3579 f_{n+4} \\
& \left.\quad-786 f_{n+5}+85 f_{n+6}\right), \\
& h y_{n+3}^{\prime}-\frac{1}{3} y_{n+3}+\frac{1}{3} y_{n}=\frac{h^{2}}{13440}\left(315 f_{n}+4590 f_{n+1}\right. \\
& \quad+9369 f_{n+2}+6576 f_{n+3}-1107 f_{n+4}+270 f_{n+5} \\
& \left.\quad-33 f_{n+6}\right),
\end{aligned}
$$

$$
\begin{aligned}
& h y_{n+4}^{\prime}-\frac{1}{3} y_{n+3}+\frac{1}{3} y_{n}=\frac{h^{2}}{120960}\left(2453 f_{n}\right. \\
& +44526 f_{n+1}+70779 f_{n+2}+135812 f_{n+3} \\
& \left.+51675 f_{n+4}-3090 f_{n+5}+245 f_{n+6}\right)
\end{aligned}
$$

$$
\begin{aligned}
& h y_{n+5}^{\prime}-\frac{1}{3} y_{n+3}+\frac{1}{3} y_{n}=\frac{h^{2}}{120960}\left(2995 f_{n}\right. \\
& \quad+40350 f_{n+1}+85377 f_{n+2}+103300 f_{n+3} \\
& \left.\quad+145653 f_{n+4}+47166 f_{n+5}-1481 f_{n+6}\right),
\end{aligned}
$$

$$
\begin{aligned}
& h y_{n+6}^{\prime}-\frac{1}{3} y_{n+3}+\frac{1}{3} y_{n}=\frac{h^{2}}{13440}\left(141 f_{n}+5886 f_{n+1}\right. \\
& \quad+4995 f_{n+2}+19812 f_{n+3}+5859 f_{n+4}+19710 f_{n+5} \\
& \left.\quad+4077 f_{n+6}\right)
\end{aligned}
$$

$$
n=0(6)(N-6) \text {. }
$$

Remark 1. We note here that, for higher orders (higher degree polynomials of (9)), the accuracy of the scheme becomes lower and tends to integrate the scheme less accurately. This is due to the truncation errors in the numerical solution.

\section{Convergence of the BVMs}

In this section, we shall establish the convergence of the BVMs derived in the previous section. We emphasize that we evaluate (6) at $x_{1}, x_{2}, \ldots, x_{v-1}, x_{v+1}, \ldots, x_{2 v}$ to obtain

$$
\begin{gathered}
y_{n+1}+\alpha_{v}^{(1)} y_{n+v}+\alpha_{0}^{(1)} y_{n}=h^{2} \sum_{i=0}^{2 v} \beta_{i}^{(1)} f_{n+i} \\
y_{n+2}+\alpha_{v}^{(2)} y_{n+v}+\alpha_{0}^{(2)} y_{n}=h^{2} \sum_{i=0}^{2 v} \beta_{i}^{(2)} f_{n+i} \\
\vdots \\
y_{n+v-1}+\alpha_{v}^{(v-1)} y_{n+v}+\alpha_{0}^{(v-1)} y_{n}=h^{2} \sum_{i=0}^{2 v} \beta_{i}^{(v-1)} f_{n+i}
\end{gathered}
$$




$$
\begin{gathered}
y_{n+v+1}+\alpha_{v}^{(v+1)} y_{n+v}+\alpha_{0}^{(v+1)} y_{n}=h^{2} \sum_{i=0}^{2 v} \beta_{i}^{(v+1)} f_{n+i} \\
\vdots \\
y_{n+2 v}+\alpha_{v}^{(2 v)} y_{n+v}+\alpha_{0}^{(2 v)} y_{n}=h^{2} \sum_{i=0}^{2 v} \beta_{i}^{(2 v)} f_{n+i}
\end{gathered}
$$

and also evaluate $U^{\prime}(x)$ at $x_{i}, \quad i=0(1)(2 v)$ to obtain

$$
\begin{gathered}
h y_{n}^{\prime}+\alpha_{v}^{\prime(0)} y_{n+v}+\alpha_{0}^{\prime(0)} y_{n}=h^{2} \sum_{i=0}^{2 v} \beta_{i}^{\prime(0)} f_{n+i}, \\
h y_{n+1}^{\prime}+\alpha_{v}^{\prime(1)} y_{n+v}+\alpha_{0}^{\prime(1)} y_{n}=h^{2} \sum_{i=0}^{2 v} \beta_{i}^{\prime(1)} f_{n+i}, \\
\vdots \\
h y_{n+2 v}^{\prime}+\alpha_{v}^{\prime(2 v)} y_{n+v}+\alpha_{0}^{\prime(2 v)} y_{n}=h^{2} \sum_{i=0}^{2 v} \beta_{i}^{\prime(2 v)} f_{n+i} .
\end{gathered}
$$

We note that the formulas in 3 are of $O\left(h^{k+4}\right)$ while the derivative formulas are of $O\left(h^{k+3}\right)$. Equations 3 and (16) can be compactly written in matrix form by introducing the following matrix notations. Let $R$ be a $2 N \times 2 N$ matrix defined by

$$
R=\left[\begin{array}{ll}
R_{11} & R_{12} \\
R_{21} & R_{22}
\end{array}\right],
$$

where $R_{i j}$ are $N \times N$ matrices given as

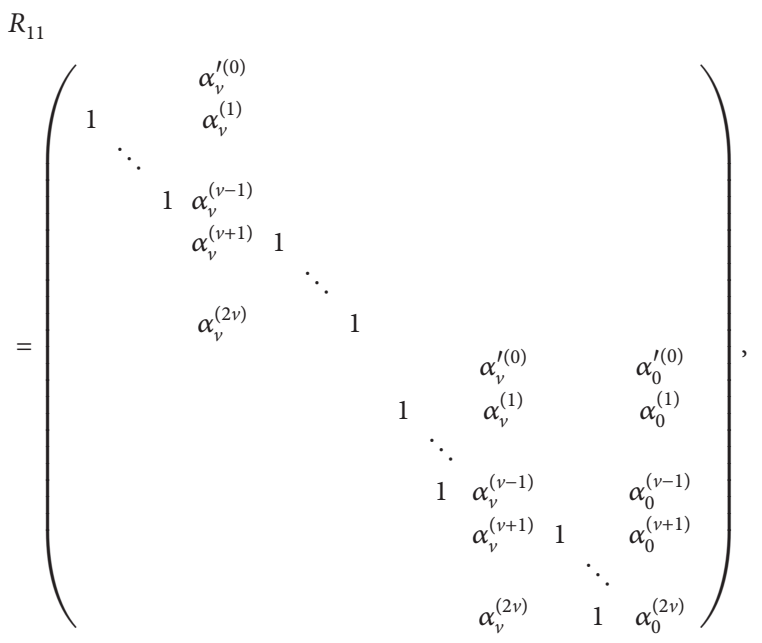

$$
R_{21}=\left(\begin{array}{ccc}
\alpha_{v}^{\prime(1)} & & \\
\vdots & & \\
\alpha_{v}^{\prime(2 v)} & & \\
& \alpha_{v}^{\prime(1)} & \alpha_{0}^{\prime(1)} \\
& \vdots & \vdots \\
& \alpha_{v}^{\prime(2 v)} & \alpha_{0}^{\prime(2 v)}
\end{array}\right),
$$

$R_{12}$ is an $N \times N$ null matrix, and $R_{22}$ is an $N \times N$ identity matrix.

Similarly, let $S$ be a $2 N \times 2 N$ matrix defined by

$$
S=\left[\begin{array}{ll}
S_{11} & S_{12} \\
S_{21} & S_{22}
\end{array}\right],
$$

where $S_{i j}$ are $N \times N$ matrices given as

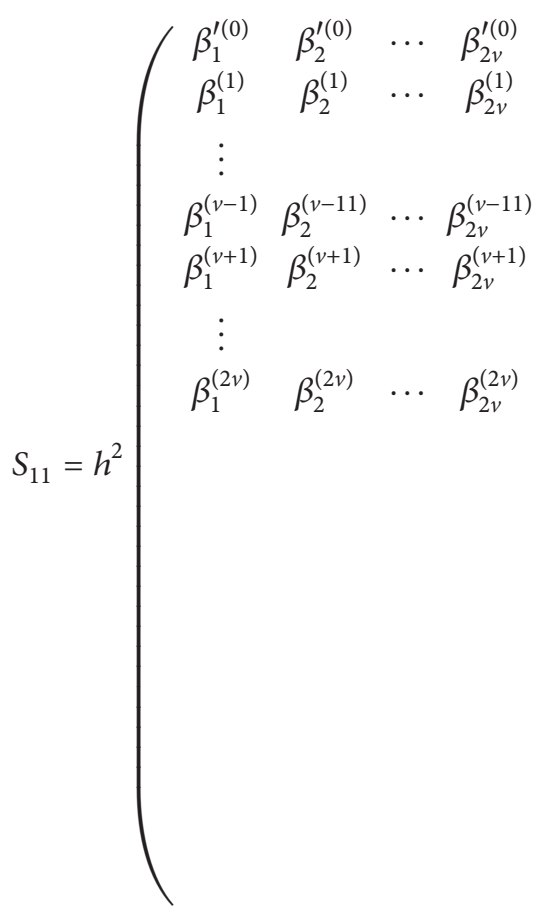

$$
\left.\begin{array}{cccccccc}
\beta_{1}^{\prime(0)} & \beta_{2}^{\prime(0)} & \cdots & \beta_{2 v}^{\prime(0)} & & & & \\
\beta_{1}^{(1)} & \beta_{2}^{(1)} & \cdots & \beta_{2 v}^{(1)} & & & & \\
\vdots & & & & & & & \\
\beta_{1}^{(v-1)} & \beta_{2}^{(v-1)} & \cdots & \beta_{2 v}^{(v-1)} & & & & \\
\beta_{1}^{(v+1)} & \beta_{2}^{(v+1)} & \cdots & \beta_{2 v}^{(v+1)} & & & & \\
\vdots & & & & & & & \\
\beta_{1}^{(2 v)} & \beta_{2}^{(2 v)} & \cdots & \beta_{2 v}^{(2 v)} & & & & \\
& & & & \ddots & \ddots & \ddots & \ddots \\
& & & & \beta_{1}^{(2 v)} & \beta_{2}^{(2 v)} & \cdots & \beta_{2 v}^{(2 v)}
\end{array}\right)
$$




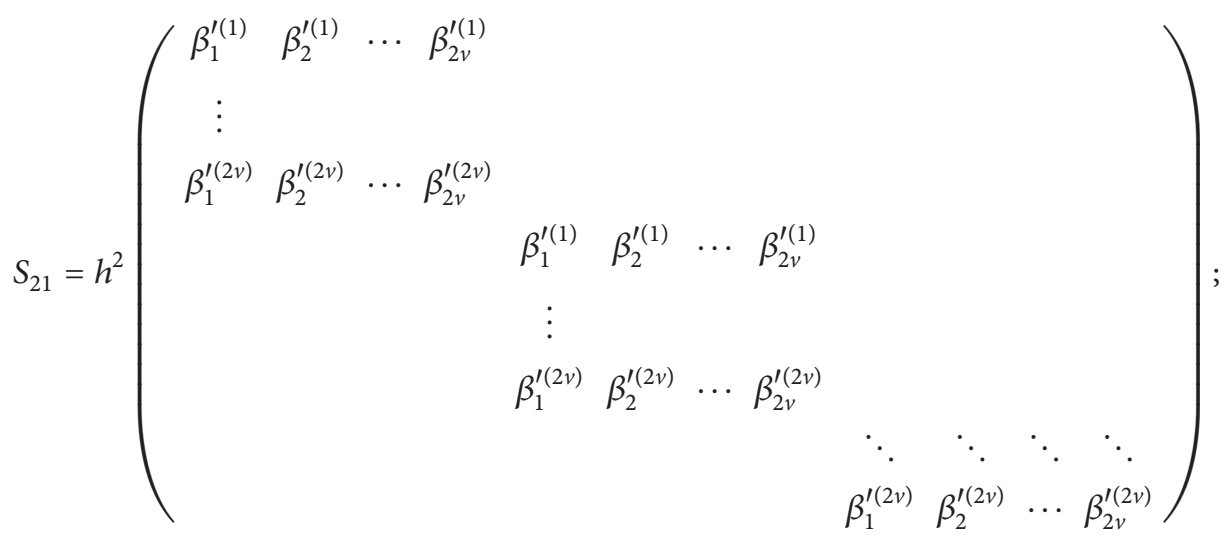

$S_{12}, S_{22}$ are $N \times N$ null matrices.

We further define the following vectors:

$$
\begin{aligned}
& \bar{Y}=\left(y_{n+1}, \ldots, y_{n+2 v}, h y_{n+1}^{\prime}, \ldots, h y_{n+2 v}^{\prime}\right)^{T}, \\
& Y=\left(y\left(x_{n+1}\right), \ldots, y\left(x_{n+2 v}\right), h y^{\prime}\left(x_{n+1}\right), \ldots,\right. \\
&\left.h y^{\prime}\left(x_{n+2 v}\right)\right)^{T}, \\
& F=\left(f_{n+1}, \ldots, f_{n+2 v}, h f_{n+1}^{\prime}, \ldots, h f_{n+2 v}^{\prime}\right)^{T}, \\
& L(h)=\left(l_{1}, \ldots, l_{N}, h l_{1}^{\prime}, \ldots, h l_{N}^{\prime}\right)^{T}, \\
& C=\left(\beta_{0}^{\prime(0)} h^{2} f_{0}-h y_{0}^{\prime}-\alpha_{0}^{\prime(0)} y_{0}, \beta_{0}^{(1)} h^{2} f_{0}-\alpha_{0}^{(1)} y_{0}, \ldots,\right. \\
& \beta_{0}^{(v-1)} h^{2} f_{0}-\alpha_{0}^{(v-1)} y_{0}, \beta_{0}^{(v+1)} h^{2} f_{0}-\alpha_{0}^{(v+1)} y_{0}, \ldots, \\
&\left.\beta_{0}^{(2 v)} h^{2} f_{0}-\alpha_{0}^{(2 v)} y_{0}, 0, \ldots, 0\right)^{T} .
\end{aligned}
$$

The exact form of the system formed by 3 and (16) is given by

$$
R Y-S F(Y)+C+L(h)=0
$$

where $L(h)$ is the truncation error vector of the formulas in 3 and (16). The approximate form of the system is given by

$$
R \bar{Y}-S F(\bar{Y})+C=0
$$

where $\bar{Y}$ is the approximate solution of vector $Y$.

Subtracting (22) from (23) and letting $E=\bar{Y}-Y=\left(e_{1}, \ldots\right.$, $\left.e_{N}, e_{1}^{\prime}, \ldots, e_{N}^{\prime}\right)^{T}$ and using the mean value theorem, we have the error system

$$
(R-S J) E=L(h),
$$

where $J$ is the Jacobian matrix and its entries $J_{11}, J_{12}, J_{21}$, and $J_{22}$ are defined as

$$
\begin{aligned}
& J_{11}=\left(\begin{array}{ccc}
\frac{\partial f_{1}}{\partial y_{1}} & \cdots & \frac{\partial f_{1}}{\partial y_{N}} \\
\vdots & \vdots & \vdots \\
\frac{\partial f_{N}}{\partial y_{1}} & \cdots & \frac{\partial f_{N}}{\partial y_{N}}
\end{array}\right), \\
& J_{12}=\left(\begin{array}{ccc}
\frac{\partial f_{1}}{\partial y_{1}^{\prime}} & \cdots & \frac{\partial f_{1}}{\partial y_{N}^{\prime}} \\
\vdots & \vdots & \vdots \\
\frac{\partial f_{N}}{\partial y_{1}^{\prime}} & \cdots & \frac{\partial f_{N}}{\partial y_{N}^{\prime}}
\end{array}\right),
\end{aligned}
$$$$
J_{21}=h\left(\begin{array}{ccc}
\frac{\partial f_{1}^{\prime}}{\partial y_{1}} & \cdots & \frac{\partial f_{1}^{\prime}}{\partial y_{N}} \\
\vdots & \vdots & \vdots \\
\frac{\partial f_{N}^{\prime}}{\partial y_{1}} & \cdots & \frac{\partial f_{N}^{\prime}}{\partial y_{N}}
\end{array}\right)
$$

$$
J_{22}=h\left(\begin{array}{ccc}
\frac{\partial f_{1}^{\prime}}{\partial y_{1}^{\prime}} & \cdots & \frac{\partial f_{1}^{\prime}}{\partial y_{N}^{\prime}} \\
\vdots & \vdots & \vdots \\
\frac{\partial f_{N}^{\prime}}{\partial y_{1}^{\prime}} & \cdots & \frac{\partial f_{N}^{\prime}}{\partial y_{N}^{\prime}}
\end{array}\right)
$$

Let $M=-S J$ be a matrix of dimension $2 N$ so that (24) becomes

$$
(R+M) E=L(h)
$$


and for sufficiently small $h, R+M$ is a monotone matrix and thus nonsingular (Jator and $\mathrm{Li}[14]$ ). Therefore

$$
\begin{aligned}
(R+M)^{-1} & =D=\left(d_{i j}\right) \geq 0, \\
\sum_{j=1}^{2 N} d_{i j} & =O\left(h^{-2}\right), \\
E & =D L(h), \\
\|E\| & =\|D L(h)\|, \\
& =O\left(h^{-2}\right) O\left(h^{k+4}\right), \\
& =O\left(h^{k+2}\right)
\end{aligned}
$$

which shows that the methods are convergent and the global error is of order $O\left(h^{k+2}\right)$.

3.1. Computational Complexities. In this section, we discuss the use of 3 and (16), which we henceforth call BVM (BVM), for $=0(2 v)(N-2 \nu)$, where $N$ is a multiple of $2 \nu$. We emphasize that the methods in the BVM are all main methods since they are weighted the same and their use lead to a single matrix equation which can be solved for the unknowns. For example, for BVM6 (order 6), we make use of each of the methods above in steps of 4 ; that is, $n=0,4, \ldots, N-4$. This results in a system of $2 N$ equations in $2 N$ unknowns which can be easily solved for the unknowns. Below is an algorithm for the use of the methods.

The methods are implemented as BVMs by efficiently using the following steps.

Step 1. Use the BVM for $n=0$ to obtain $\mathbf{Y}_{1}$ in the interval $\left[y_{0}, y_{2 v}\right]$ and for $n=1 \mathbf{Y}_{2}$ is obtained in the interval $\left[y_{2 v}, y_{4 v}\right]$. Similarly, for $n=2,3, \ldots,(\Gamma-1)$ we obtain $\mathbf{Y}_{3}, \ldots, \mathbf{Y}_{\Gamma}$, where $N=2 v \times \Gamma$ in the intervals, $\left[y_{4 v}, y_{6 v}\right],\left[y_{6 v}, y_{8 v}\right], \ldots,\left[y_{N-2 v}, y_{N}\right]$, respectively.

Step 2. The unified block given by the system $\mathbf{Y}_{1} \cup \mathbf{Y}_{2} \cup \cdots \cup$ $\mathbf{Y}_{\Gamma-1} \cup \mathbf{Y}_{\Gamma}$ obtained in Step 1 results in a system of $2 N$ equations in $2 \mathrm{~N}$ unknowns which can be easily solved.

Step 3. The values of the solution and the first derivatives of (1) are generated by the sequence $\left\{y_{n}\right\},\left\{y_{n}^{\prime}\right\}, n=0, \ldots, N$, obtained as the solution in Step 2.

\section{Lanczos-Chebyshev Reduction Technique}

We consider the second-order problem of the form

$$
\mathbf{L} u(x, y)=f \quad \text { in } \Upsilon
$$

with the boundary condition

$$
\mathbf{L} u(x, y)=g \quad \text { on } \Omega,
$$

where $\Omega$ is the boundary of the region $\Upsilon$. The Lanczos $\tau$ method involves the replacement of one of the two functions in the trial solution by an approximate polynomial of the form

$$
G_{i}=x^{i-1}
$$

and $u(x, y)$ is approximated by

$$
u(x, y) \approx u_{N}(x, y)=\sum_{i=1}^{N} p_{i}(y) x^{i-1}
$$

and $\Omega$ must be bounded by the lines $x= \pm 1$ in the $x$ direction. The problem is slightly perturbed to obtain

$$
\begin{aligned}
& \mathbf{L} u_{N}(x, y)=\tau_{1}(y) C_{N-2}^{*}(x)+\tau_{2}(y) C_{N-1}^{*}(x)+f \\
& \mathbf{L} u_{N}(x, y)=g, \quad x \in[a, b]
\end{aligned}
$$

where $\tau_{i}(y)$ are arbitrary functions and $C_{j}^{*}(x)$ are the $j$ th order shifted Chebyshev polynomials in the range $x \in$ $[a, b]$. Equating the powers of $x$ in (32) together with the boundary conditions (33) gives $(N+2)$ equations in $(N+2)$ unknowns $\tau_{1}(y), \tau_{2}(y), p_{1}(y), \ldots, p_{N}(y)$. The operator $\mathbf{L}$ may also contain polynomial functions. The inclusion of (33) is to ensure the exact satisfaction of the exact solutions at $x=a$ and $x=b$. The arbitrary $\tau$ functions are eliminated to give a set of $N$ second-order ordinary differential equations. Hence, $p_{i}(y)$ can be uniquely determined.

\section{Numerical Illustrations}

In this section, we consider five numerical examples using the BVMs of order 6 to solve the ODE system arising from the semidiscretization of the PDEs. All computations were carried out using a written code in Mathematica 11.0. In all examples, a uniform stepsize was used and the maximum absolute errors were computed as

$$
\max \left\|\left(u_{i j}-u\left(x_{i}, y_{j}\right)\right)\right\|, \quad 0 \leq i \leq N, 0 \leq j \leq M,
$$

where $u_{i j}$ is the numerical approximation of the exact solution $u\left(x_{i}, y_{j}\right)$ at the mesh point $\left(x_{i}, y_{j}\right)$.

Example 1. We consider the following one-dimensional elliptic PDE:

$$
\begin{aligned}
& u_{x x}+u_{y y}=2\left(x^{2}+y^{2}-2\right), \quad x, y \in[-1,1], \\
& u(x, \pm 1)=0
\end{aligned}
$$

Exact: $u(x, y)=\left(x^{2}-1\right)\left(y^{2}-1\right)$.

We seek an approximation of the form

$$
u_{N}(x, y)=\sum_{i=1}^{N} p_{i}(y) x^{i-1}
$$

to obtain

$$
\nabla^{2} u=\sum_{i=1}^{N}\left\{p_{i}^{\prime \prime}(y)+i(i+1) p_{i+2}(y)\right\} x^{i-1}
$$

and the perturbed form

$$
\begin{aligned}
\nabla^{2} u_{N}(x, y)= & 2\left(x^{2}+y^{2}-2\right)+\tau_{1} C_{N-2}(x) \\
& +\tau_{2}(y) C_{N-1}(x)
\end{aligned}
$$


TABLE 1: Maximum errors on the line $x=0$ for the elliptic PDE in Example 1 .

\begin{tabular}{ccccc}
\hline \multicolumn{5}{c}{$N$} \\
4 & 5 & 6 & 7 & 8 \\
\hline $4.441 e-16$ & $2.776 e-16$ & $2.220 e-16$ & $2.43 e-16$ & $2.220 e-16$ \\
\hline
\end{tabular}

with

$$
\nabla^{2} u_{N}(x, y)=\left.2\left(x^{2}+y^{2}-2\right)\right|_{x= \pm 1},
$$

where

$$
C_{N}(x)=\sum_{i=1}^{N} C_{i}^{N} x^{i}, \quad-1 \leq x \leq 1 .
$$

Equating the powers of $x$ in (38), we obtain

$$
\begin{aligned}
& p_{i}^{\prime \prime}(y)+i(i+1) p_{i+2}(y) \\
& =\tau_{1}(y) C_{i-1}^{N-2}+\tau_{2}(y) C_{i-1}^{N-1} \\
& i=2,4,5, \ldots, N-2, \\
& p_{1}^{\prime \prime}(y)+2 p_{3}(y) \\
& =-4+2 y^{2}+\tau_{1}(y) C_{0}^{N-2}+\tau_{2}(y) C_{0}^{N-1}, \\
& p_{3}^{\prime \prime}(y)+12 p_{5}(y)=2+\tau_{1}(y) C_{2}^{N-2}+\tau_{2}(y) C_{2}^{N-1} \text {, } \\
& p_{N-1}^{\prime \prime}(y)=\tau_{1}(y) C_{N-2}^{N-2}+\tau_{2}(y) C_{N-2}^{N-1}, \\
& p_{N}^{\prime \prime}(y)=\tau_{2}(y) C_{N-1}^{N-1} \text {. }
\end{aligned}
$$

From (39) and (41), we eliminate $\tau_{i}(y)$ to obtain $N$ secondorder differential equations in $N$ unknowns $p_{i}(y), i=$ 1(1)N. To use the BVMs to solve the resulting second-order system, we require $N$ boundary conditions which is obtained from $u(x, \pm 1)=0$; that is, $p_{0}( \pm 1)=\cdots=p_{N}( \pm 1)=0$. Table 1 shows the computational results; Figures $1-3$ show the exact, approximate, and error function of the elliptic problem, respectively.

Example 2. We consider the one-dimensional wave equation with variable coefficients

$$
\begin{aligned}
\frac{x^{2}}{2} u_{x x}-u_{y y} & =0, \quad x, y \in[0,1], \\
u(x, 0) & =x, \\
u_{y}(x, 0) & =x^{2}
\end{aligned}
$$

Exact: $u(x, y)=x+x^{2} \sinh (y)$.

The perturbed form of the problem is

$$
\begin{aligned}
\frac{x^{2}}{2}\left(u_{N}\right)_{x x}-\left(u_{N}\right)_{y y}= & \tau_{1}(y) C_{N-2}^{*}(x) \\
& +\tau_{2}(y) C_{N-1}^{*}(x)
\end{aligned}
$$

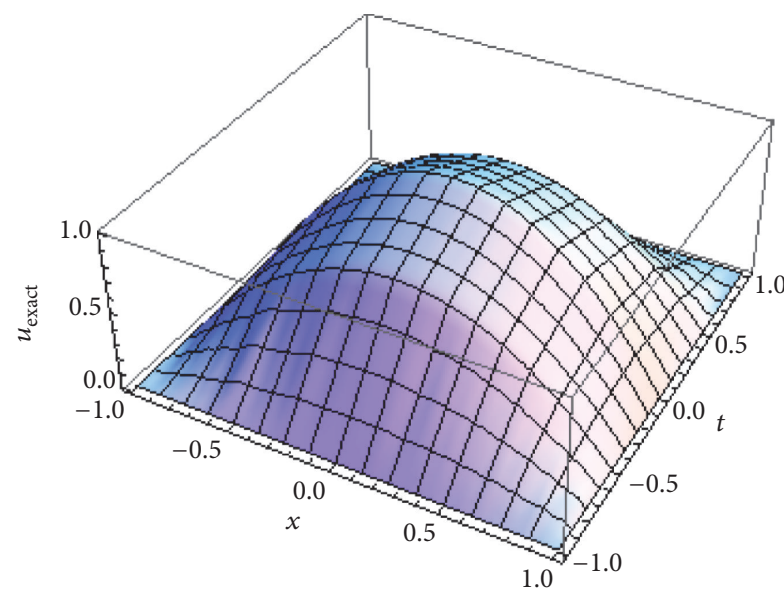

Figure 1: Graph of the exact solution for Example 1.

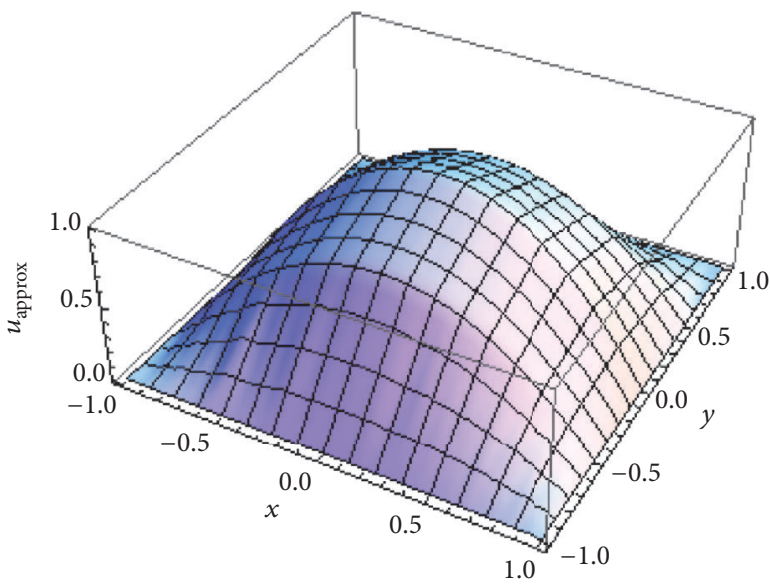

FIgURE 2: Graph of the approximate solution for Example 1.

with

$$
\begin{aligned}
\frac{x^{2}}{2}\left(u_{N}\right)_{x x}-\left(u_{N}\right)_{y y} & =\left.0\right|_{x=0,1} \\
C_{N}^{*}(x) & =\sum_{i=0}^{N} C_{i}^{N} x^{i}, \quad 0 \leq x \leq 1 .
\end{aligned}
$$

Equating powers of $x$, we obtain

$$
\begin{aligned}
& \frac{1}{2}(i-1)(i+1) p_{i}(y)-p_{i}^{\prime \prime}(y) \\
& \quad=\tau_{1}(y) C_{i-1}^{N-2}+\tau_{2}(y) C_{i-1}^{N-1} \quad i=3, \ldots, N-1, \\
& p_{1}^{\prime \prime}(y)=-\tau_{1}(y) C_{0}^{N-2}-\tau_{2}(y) C_{0}^{N-1}, \\
& p_{2}^{\prime \prime}(y)=-\tau_{1}(y) C_{1}^{N-2}-\tau_{2}(y) C_{1}^{N-1}, \\
& p_{N}^{\prime \prime}(y)=-\tau_{2}(y) C_{N-1}^{N-1} .
\end{aligned}
$$

Eliminating $\tau_{i}(y)$ from (44) and (45), we obtain $N$ secondorder differential equations in $N$ unknowns $p_{i}(y), i=1(1) N$. 


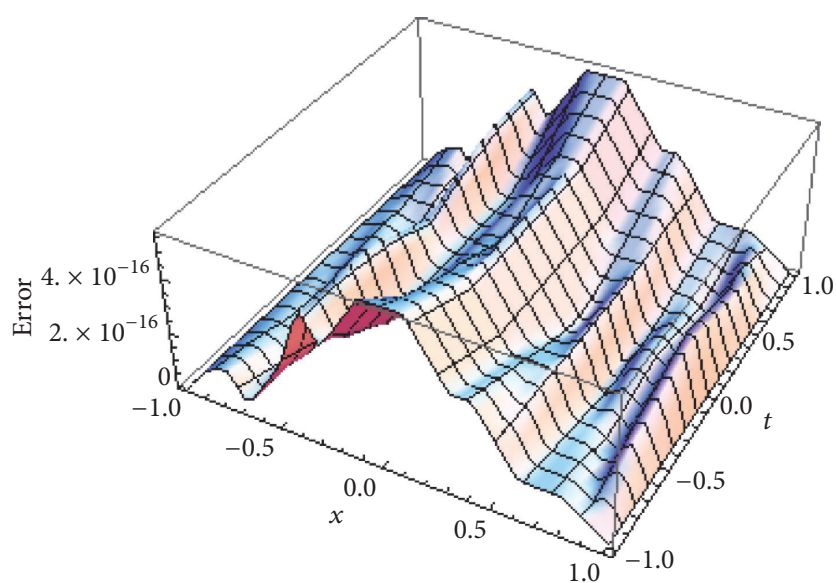

FIGURE 3: Graph of the error function for Example 1.

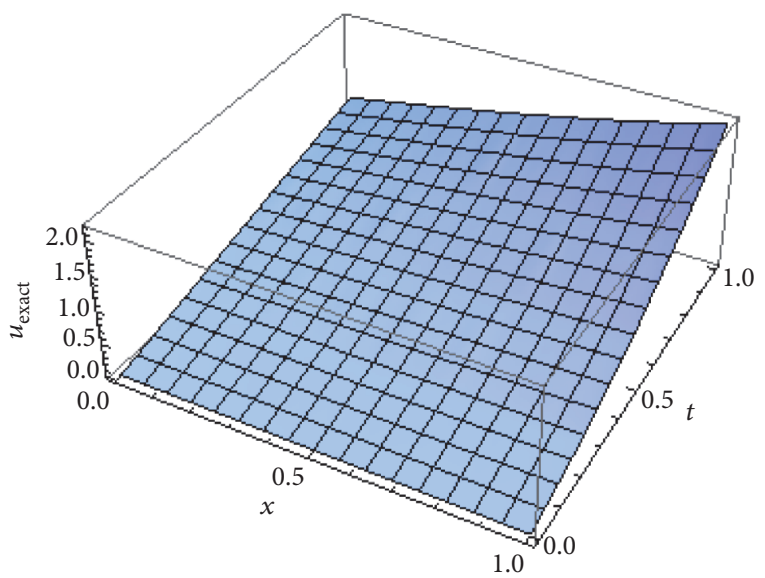

FIGURE 4: Graph of the exact solution for Example 2.

The initial conditions to be used with this system of secondorder ODE are

$$
\begin{aligned}
& p_{1}(0)=0, \\
& p_{2}(0)=1, \\
& p_{3}(0)=\cdots=p_{N}(0)=0, \\
& p_{1}^{\prime}(0)=0 \\
& p_{2}^{\prime}(0)=0 \\
& p_{3}^{\prime}(0)=1, \\
& p_{4}^{\prime}(0)=\cdots=p_{N}^{\prime}(0)=0 .
\end{aligned}
$$

Table 2 shows the maximum errors on the line $x=0.5$ for different $N$. Also Figures 4-6 depict the exact, approximate, and error function of the wave equation.

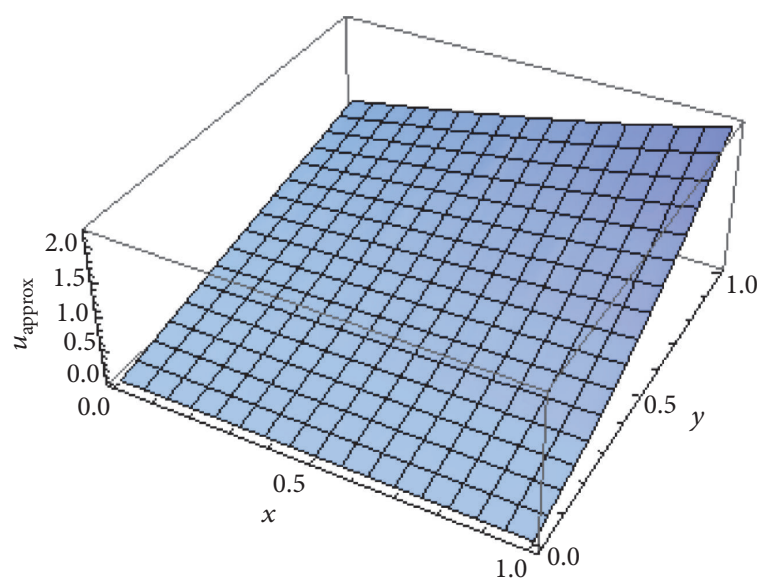

FIGURE 5: Graph of the approximate solution for Example 2.

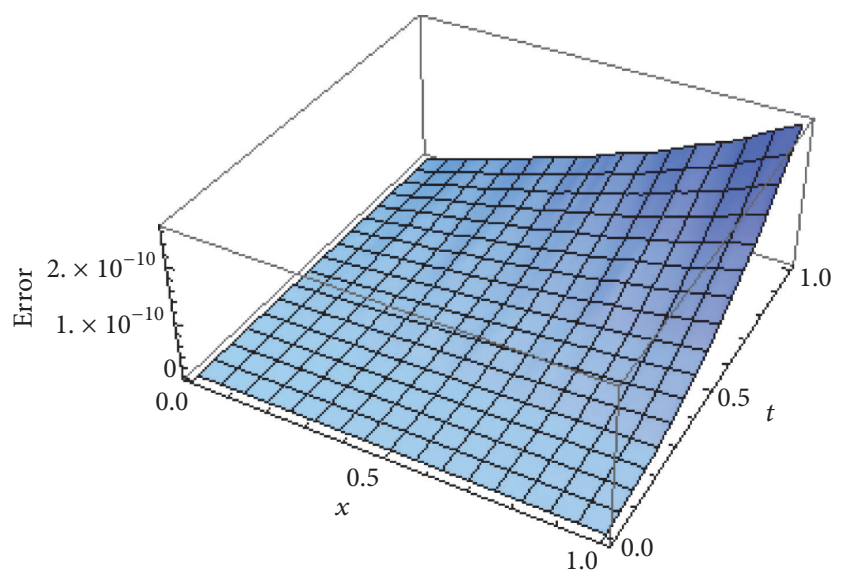

FIgURE 6: Graph of the error function for Example 2.

TABLE 2: Maximum errors on the line $x=0.5$ for the wave equation in Example 2.

\begin{tabular}{ccccc}
\hline \multicolumn{5}{c}{$N$} \\
4 & 5 & 6 & 7 & 8 \\
\hline $6.579 e-11$ & $4.211 e-11$ & $2.924 e-11$ & $2.148 e-11$ & $1.645 e-11$ \\
\hline
\end{tabular}

Example 3. Consider the one-dimensional linear inhomogeneous Klein-Gordon equation

$$
\begin{aligned}
u_{t t}-u_{x x}+u & =6 x^{3} t+t^{3}\left(x^{3}-6 x\right), \quad x, t \in[0,1] \\
u(x, 0) & =0 \\
u_{t}(x, 0) & =0
\end{aligned}
$$

Exact: $u(x, t)=x^{3} t^{3}$.

With the procedures of the previous examples, we obtain the maximum errors as shown in Table 3. Figures 7-9 also show the exact, approximate, and error function of the KleinGordon equation. 


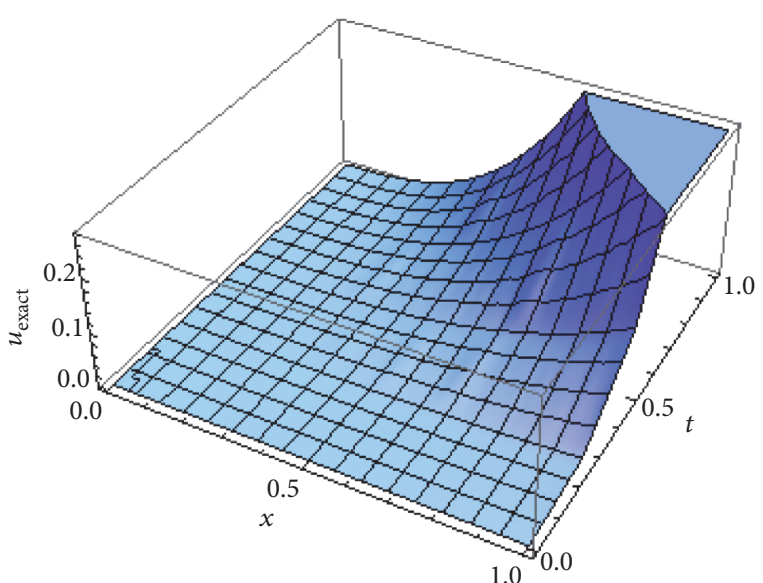

Figure 7: Graph of the exact solution for Example 3.

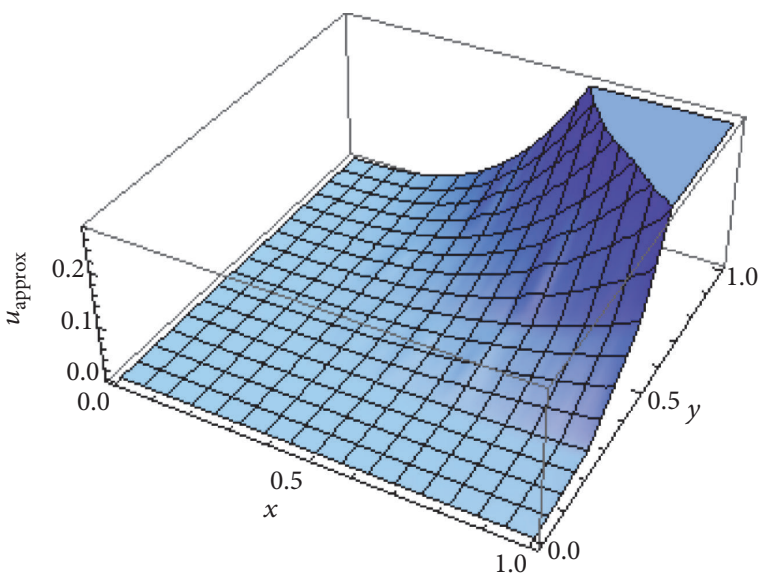

FIGURE 8: Graph of the approximate solution for Example 3.

TABLe 3: Maximum errors on the line $x=0.5$ for the Klein-Gordon equation in Example 3.

\begin{tabular}{ccccc}
\hline \multicolumn{5}{c}{$N$} \\
4 & 5 & 6 & 7 & 8 \\
\hline $4.163 e-17$ & $1.388 e-17$ & $6.939 e-18$ & $3.469 e-18$ & $3.469 e-18$
\end{tabular}

Example 4. Consider the Helmholtz equation

$$
\begin{aligned}
u_{x x}+u_{y y}-u & =0, \quad x, y \in[0,1], \\
u(x, 0) & =0, \\
u_{y}(x, 0) & =e^{x}
\end{aligned}
$$$$
\text { Exact: } u(x, y)=y e^{x}+x \cosh (y) \text {. }
$$

Table 4 shows the numerical results for the Helmholtz equation. We observe that, for this example, as $N$ increases the method gives more accurate results. This is due to the accurate matching of the derivative conditions as $N$ increases. Figures 10-12 show the plot of the exact, approximate, and error function for this example.

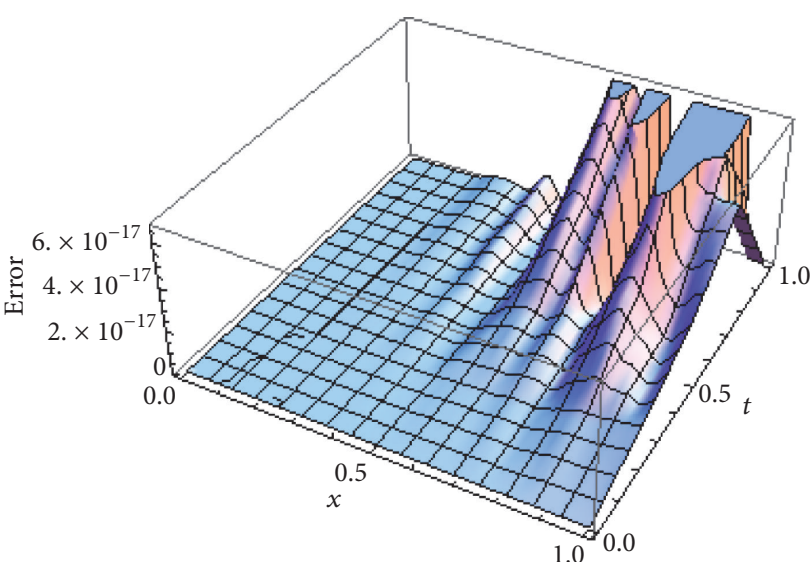

FIgURE 9: Graph of the error function for Example 3.

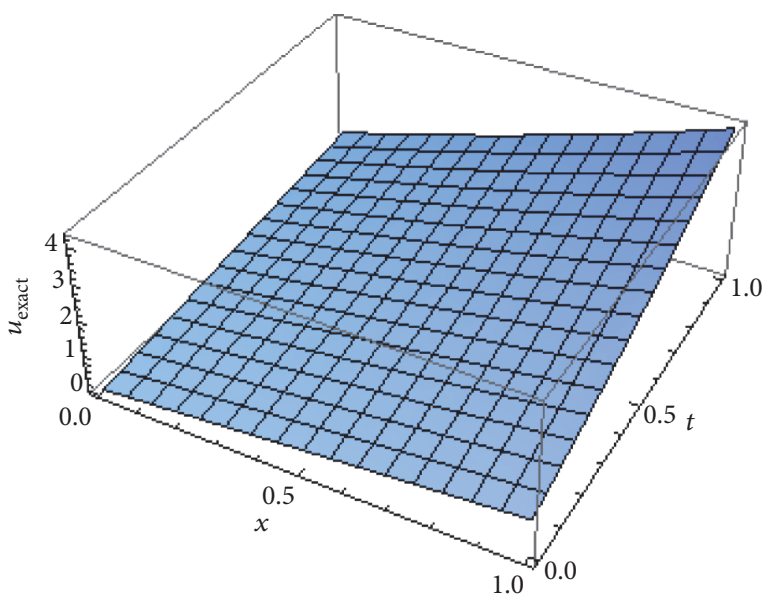

FIGURE 10: Graph of the exact solution for Example 4.

TABle 4: Maximum errors on the line $x=0.5$ for the Helmholtz equation in Example 4.

\begin{tabular}{cccc}
\hline \multicolumn{4}{c}{$N$} \\
4 & 6 & 8 & 10 \\
\hline $9.554 e-03$ & $.170 e-04$ & $1.998 e-06$ & $1.639 e-08$ \\
\hline
\end{tabular}

Example 5. Lastly, we consider the nonlinear equation

$$
\begin{aligned}
u_{t t} & =\left(u u_{x}\right)_{x}, \quad x, t \in[0,1], \\
u(x, 0) & =x^{2} \\
u_{t}(x, 0) & =-2 x^{2} \\
\text { Exact: } u(x, t) & =\left(\frac{x}{t+1}\right)^{2} .
\end{aligned}
$$

Table 5 and Figures 13-15 show the numerical results for the nonlinear equation. 


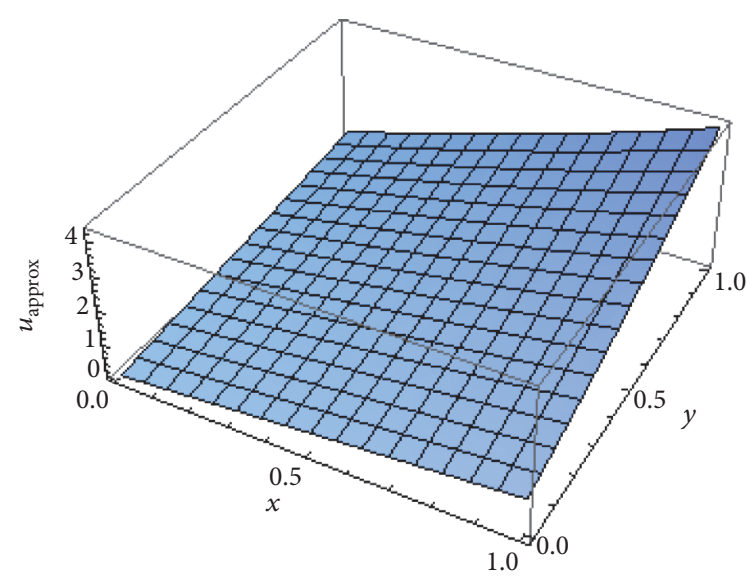

FIGURE 11: Graph of the approximate solution for Example 4.

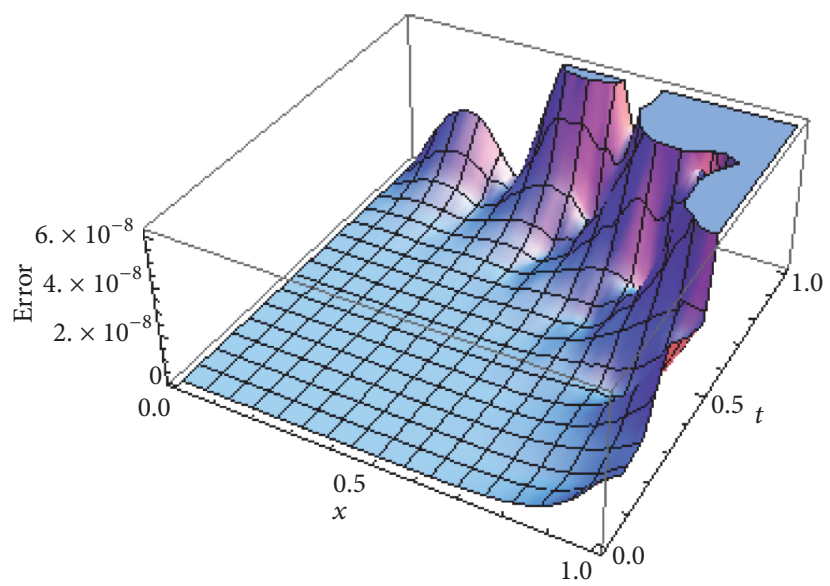

FIGURE 12: Graph of the error function for Example 4.

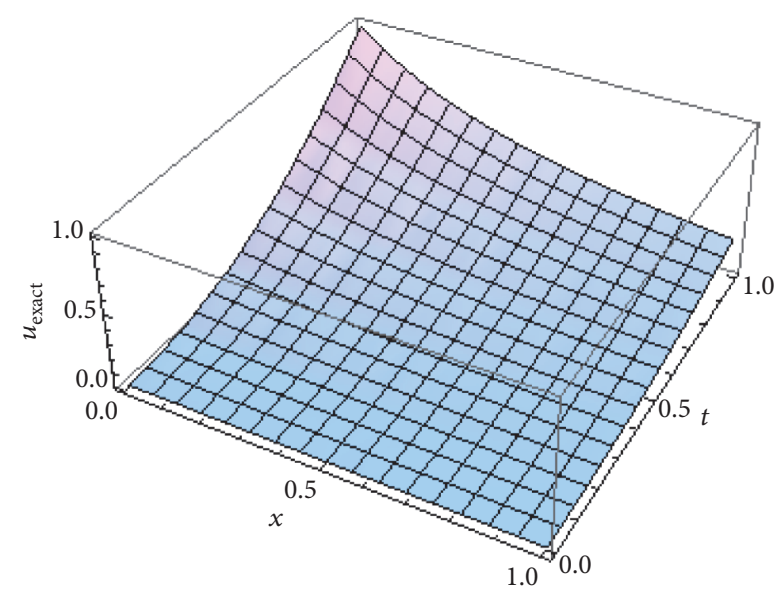

FIGURE 13: Graph of the exact solution for Example 5.

\section{Conclusion}

The Boundary Value Methods have been used to approximate second-order PDEs. This has been achieved by using the Lanczos-Chebyshev reduction technique to transform the PDEs into an equivalent second-order system. The high

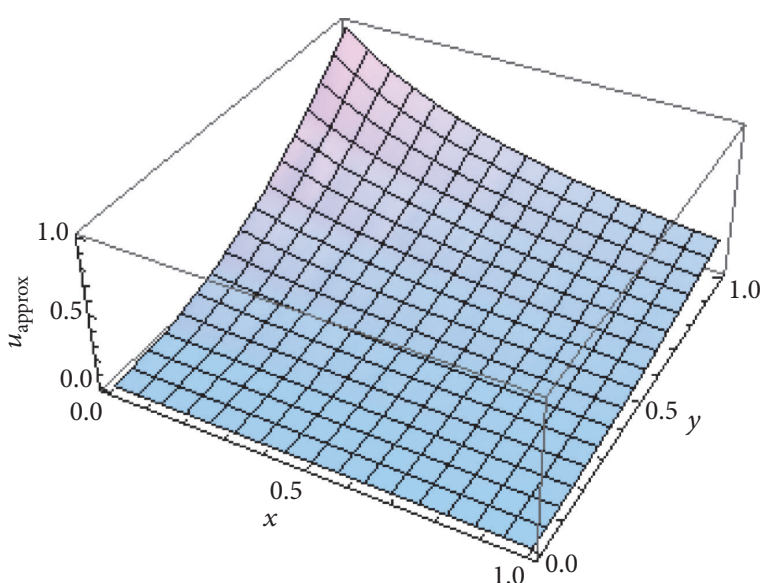

FIGURE 14: Graph of the approximate solution for Example 5.

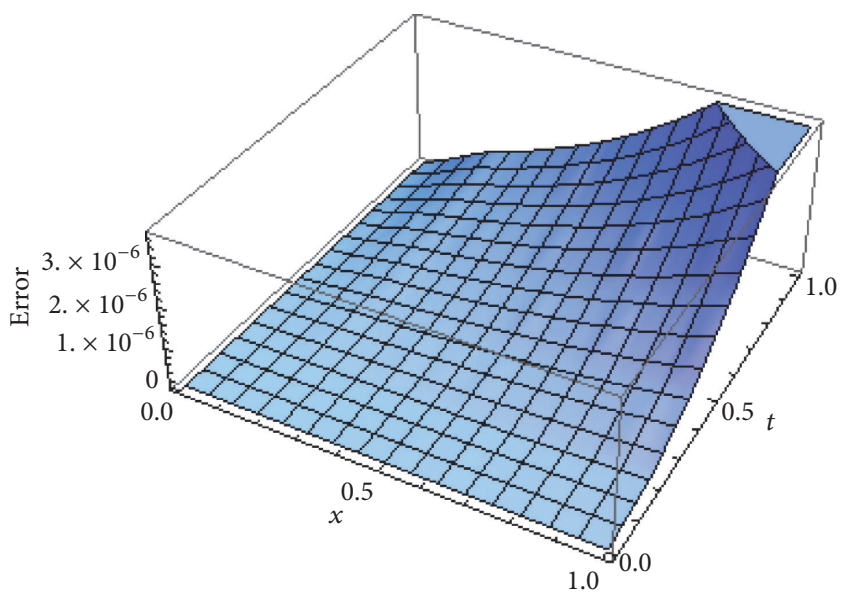

FIgURE 15: Graph of the error function for Example 5.

TABLE 5: Maximum errors on the line $x=0.5$ for the nonlinear equation in Example 5.

\begin{tabular}{ccc}
\hline & $N$ & \\
4 & & 5 \\
\hline $1.454 e-06$ & $9.304 e-07$ \\
\hline
\end{tabular}

accuracy and simplicity of the approach is evidenced by the preceding examples.

\section{Competing Interests}

The authors declare that there is no conflict of interests regarding the publication of this article.

\section{References}

[1] P. Y. P. Chen, "Stress analysis for end effects in cylindrical rods by the lanczos-chebyshev method," Nuclear Engineering and Design, vol. 52, no. 2, pp. 225-234, 1979.

[2] P. Y. Chen, "Solutions of boundary value problems by the Lanczos-Chebyshev reduction method," International Journal 
for Numerical Methods in Engineering, vol. 17, no. 2, pp. 291-299, 1981.

[3] L. Brugnano and D. Trigiante, "High-order multistep methods for boundary value problems," Applied Numerical Mathematics, vol. 18, no. 1-3, pp. 79-94, 1995.

[4] L. Brugnano and D. Trigiante, Solving Differential Problems by Multistep Initial and Boundary Value Problems, vol. 6 of Stability and Control: Theory, Methods and Applications, Gordon and Breach Science, Amsterdam, The Netherlands, 1998.

[5] L. Brugnano and D. Trigiante, "Stability properties of some boundary value methods," Applied Numerical Mathematics. An IMACS Journal, vol. 13, no. 4, pp. 291-304, 1993.

[6] P. Amodio and F. Iavernaro, "Symmetric boundary value methods for second order initial and boundary value problems," Mediterranean Journal of Mathematics, vol. 3, no. 3-4, pp. 383398, 2006.

[7] P. Amodio and F. Mazzia, "A boundary value approach to the numerical solution of initial value problems by multistep methods," Journal of Difference Equations and Applications, vol. 1, no. 4, pp. 353-367, 1995.

[8] P. Amodio and L. Brugnano, "Parallel implementation of block boundary value methods for ODEs," Journal of Computational and Applied Mathematics, vol. 78, no. 2, pp. 197-211, 1997.

[9] T. A. Biala, "A computational study of the boundary value methods and the block unification methods for $y^{\prime \prime}=f\left(x, y, y^{\prime}\right)$," Abstract and Applied Analysis, vol. 2016, Article ID 8465103, 14 pages, 2016.

[10] T. A. Biala and S. N. Jator, "A boundary value approach for solving three-dimensional elliptic and hyperbolic partial differential equations," SpringerPlus, vol. 4, no. 1, article 588, pp. 1-16, 2015.

[11] T. A. Biala, S. N. Jator, and R. B. Adeniyi, "Numerical approximations of second order PDEs by boundary value methods and the method of lines," Afrika Matematika, 2016.

[12] T. A. Biala and S. N. Jator, "A family of boundary value methods for systems of second order boundary value problems," International Journal of Differential Equations, vol. 2017, Article ID 2464759, 12 pages, 2017.

[13] P. L. Ndukum, T. A. Biala, S. N. Jator, and R. B. Adeniyi, "On a family of trigonometrically fitted extended backward differentiation formulas for stiff and oscillatory initial value problems," Numerical Algorithms, vol. 74, no. 1, pp. 267-287, 2017.

[14] S. N. Jator and J. Li, "An algorithm for second order initial and boundary value problems with an automatic error estimate based on a third derivative method," Numerical Algorithms, vol. 59, no. 3, pp. 333-346, 2012.

[15] P. Onumanyi, U. W. Sirisena, and S. N. Jator, "Continuous finite difference approximations for solving differential equations," International Journal of Computer Mathematics, vol. 72, no. 1, pp. 15-27, 1999. 


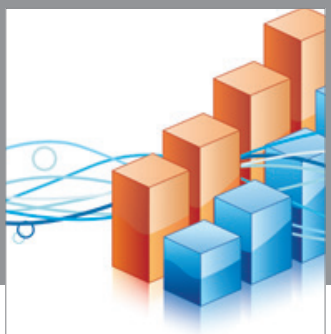

Advances in

Operations Research

vatem alat4

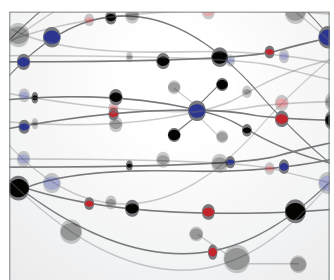

\section{The Scientific} World Journal
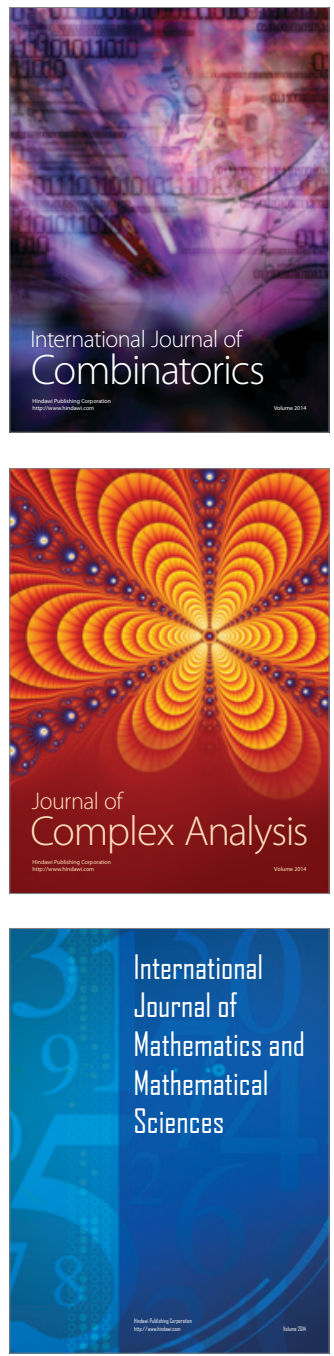
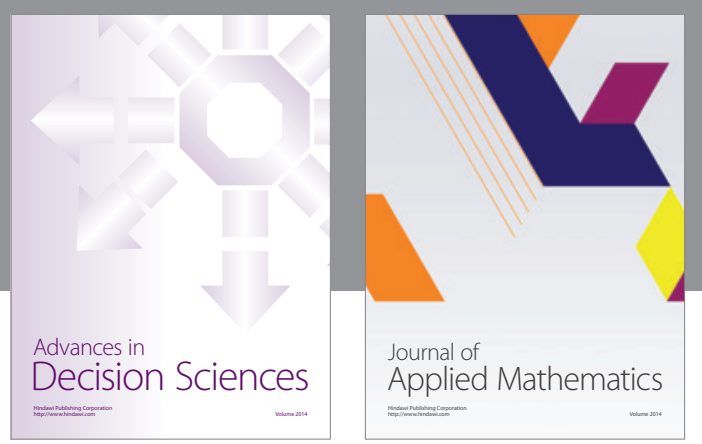

Algebra

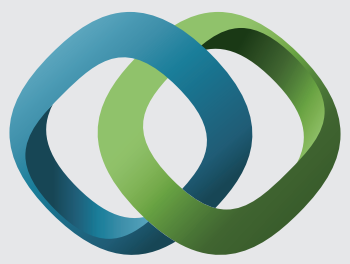

\section{Hindawi}

Submit your manuscripts at

https://www.hindawi.com
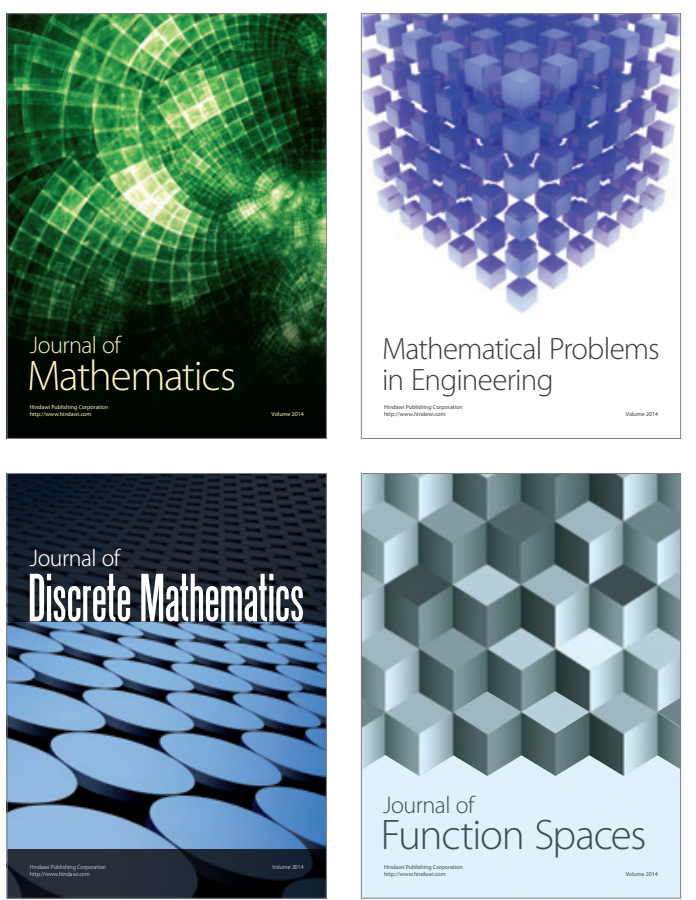

Mathematical Problems in Engineering
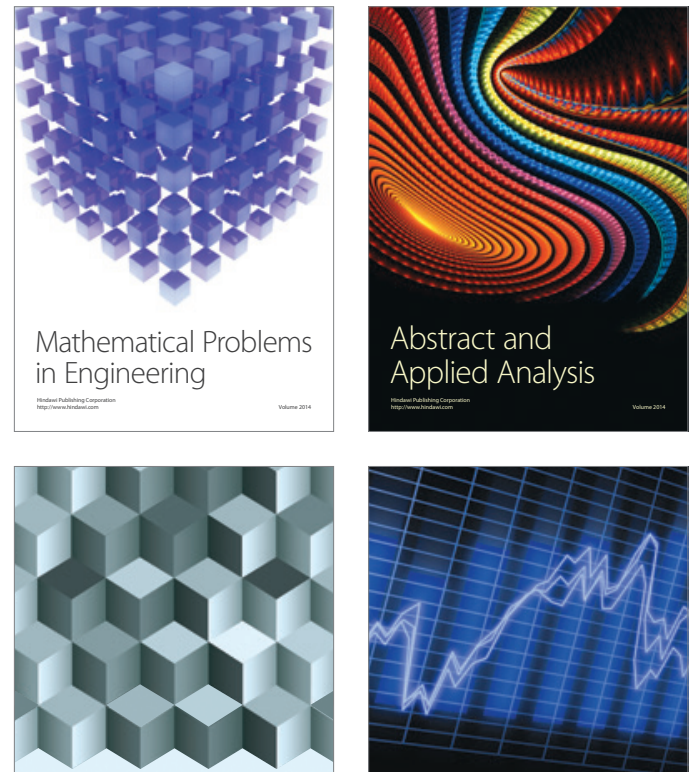

Journal of

Function Spaces

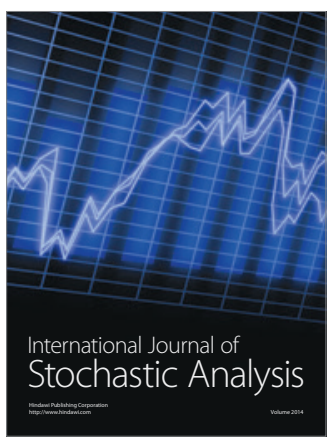

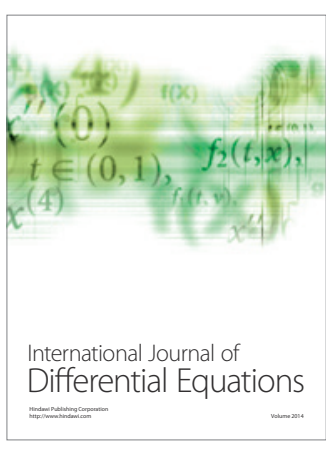
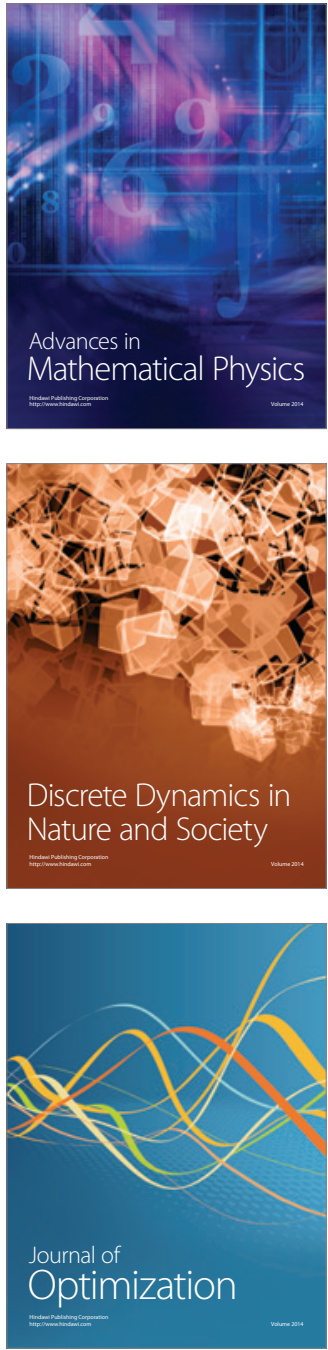